\title{
New Dimensions in Gompertzian Growth
}

\author{
ŽELJKO BAJZER ${ }^{a, b, *}$ and STANIMIR VUK-PAVLOVIĆc ${ }^{b, c}$ \\ ${ }^{a}$ Biomathematics Resource Core, ${ }^{b}$ Department of Biochemistry and Molecular Biology, ${ }^{\mathrm{c}}$ Stem Cell Laboratory, Mayo Clinic \\ Comprehensive Cancer Center, Mayo Clinic and Mayo Foundation, Rochester, MN 55905, USA
}

(Received 13 December 1998; In final form 25 February 1999)

\begin{abstract}
The Gompertz function was formulated to represent an actuarial curve, yet it often fits growth of organisms, organs and tumors. Despite numerous attempts, no consensus has been forged about the biological foundation of the broad applicability of the model. Here we revisit the Gompertzian notion of the "power to grow" and equate it with growth fraction. Aside from conferring biological interpretability to the model, this approach allows a conceptual separation of the growth fraction term from the kinetic term in the model, leading to the possibility of exploring the behavior of Gompertzian growth with fractal kinetics. Significantly, we found that empirical models such as the logistic model, the von Bertalanffy model and the von Bertalanffy-Richards model, together with the originative Gompertz model, are special cases of Gompertzian growth in fractal space. This finding permits an analysis of the growth kinetics of tumors which might affect model-based design of chemotherapy protocols.
\end{abstract}

Keywords: Growth, Gompertz formula, growth fraction, fractal kinetics, tumors

\section{INTRODUCTION}

Most of the 20th century saw the attempts to understand the kinetics of tumor growth through efforts to decide which of the numerous proposed tumor growth models "fits the growth data best". The major impetus for these attempts has been the need for a quantitative description of tumor growth ( $c f$. SimpsonHerren and Lloyd, 1970; Steel, 1977; Wheldon, 1988; Asachenkov et al., 1994), for the understanding of basic mechanisms regulating growth (cf. Bajzer et al., 1984; Bajzer and Vuk-Pavlović, 1990; Michelson and Leith, 1996; Adam and Bellomo 1997), and for prediction of tumor response to therapy ( $c f$. Sullivan and Salmon, 1972; Martin et al., 1990; Swan, 1990; Webb, 1992; Panetta, 1996, Cameron, 1997). The simplest models included nothing more than a term to encompass tumor growth (increase in volume, mass, or cellularity due to cell division) and a term to account for the loss of volume, mass, or cellularity by cell death and shedding. In a recent classification of deterministic and phenomenological models of tumor growth, we termed these models "empirical" (Marušić et al., 1994a). The two terms in empirical models, one describing tumor gain and the other tumor loss, are either based on simple biological assumptions or no assumption at all. The model by von Bertalanffy is an example of simple assumptions - gain

\footnotetext{
${ }^{*}$ Corresponding Author: Tel: 507284 8584; Fax: 507284 9420; E-mail: bajzer@mayo.edu
} 
in tumor mass proportional to tumor surface and loss of tumor mass proportional to tumor volume (von Bertalanffy, 1957; Vaidya and Alexandro, 1982). The model applied most broadly and most successfully in fitting to experimental and clinical data comprises virtually no assumptions applicable to tumors - the well known Gompertz model originally developed as an actuarial curve for the population of England almost two centuries ago (Gompertz, 1825).

In the past, the field of mathematical modeling of tumor growth was relegated mostly to a rather special community of mathematicians, experimentalists and clinicians. Nonetheless, in the last decade the field became re-energized by the always increasing advent of experimental and clinical data. Particularly important have been advances in noninvasive medical imaging (mammography, fluorography, magnetic resonance imaging, computerized tomography, ultrasound, etc.) with their high resolution that allows detection and follow-up of ever smaller tumors. Application of these techniques to population screening continues to amass large sets of data, admittedly still volumetric, that nevertheless contain a wealth of biomedical information waiting to be decoded by the application of mathematical models.

Innovations in technology and data quality have been accompanied by fresh ideas about application of empirical mathematical models to the understanding of tumor growth with the possible use in design and optimization of clinical protocols (Shochat et al., 1998). Initially, these models were applied in chemotherapy ( $c f$. Swan, 1990; Martin et al., 1990), but more recently there are new approaches. Here, understanding the kinetics of tumor growth promises optimization of time-dependent chemotherapy regimens that are more effective in eradication of tumors an less toxic to vital functions, particularly hematopoiesis (Cojocaru and Agur, 1992; Agur et al., 1992; Agur, 1998). The promise for application in cancer therapy begets the field a new prominence, but also the expectations of better mathematical insight, numerical reliability and robustness, biologic foundation and, particularly, interpretability. It is ironic that the oldest and arguably the most often applied mathematical model of tumor growth, the Gomperz model, is in want for a biological foundation as the basis for meaningful and applicable interpretation. It is no wonder that the quest for this foundation for Gompertzian growth has been a coveted goal of many over the decades.

A century after publication, the Gompertz law was proposed as a model for biological growth (Weymouth et al., 1931; Winsor, 1932) and has been studied since in numerous theoretical and applied papers dealing with organisms (e.g., Laird et al., 1965; Laird, 1966; Wosilait et al., 1992; Begall, 1997) and tumors (e.g., Laird, 1964; Durbin et al., 1967; SimpsonHerren and Loyd, 1970; Sullivan and Salmon, 1972; Norton et al., 1976; Gratton et al., 1978; Akanuma, 1978; Michelson et al., 1987; Norton, 1988; Bassukas and Maurer-Schultze, 1988; Bassukas, 1994a, 1994b; Marušić et al., 1994a, 1994b, Olea et al., 1994; Parfitt and Fyhrie, 1997). The success of the model in describing growth of biological systems prompted the question whether there is in Gompertzian growth something inherently biological, an implied assumption that underlies the ability of the formula to conform to the measured curves of biological growth.

Recently, we reviewed attempts to derive the model from some basic principles ( $c f$. Bajzer et al., 1997). These attempts included derivations within the framework of "synergistic and saturable systems" (Savageau, 1979), cell kinetics (Frenzen and Murray, 1986; Gyllenberg and Webb, 1989), and "senescence in biological hierarchies" (Witten, 1985). In these and other efforts (Xu, 1987; Makany 1991; Bajzer et al., 1996), the Gompertz formula was derived or approximated as a special case of more general models. However, in each study at least one assumption was not more biologically founded than the Gompertz formula itself (Bajzer et al., 1997). The argument of entropy change was used for a derivation of asymptotic Gompertzian growth (Calderon and Kwembe, 1991), but the assumptions were not fully justified (Bajzer et al., 1997). Another entropy-based argument led to a predicted advantage of the Gompertz model over the logistic model and the von Bertalanffy model (Ling and He, 1993), but the prediction has not been tested experimentally (Bajzer et $a l$., 1997). An attempt to derive Gompertzian growth 
from cell population heterogeneity and ideas from statistical mechanics (Kendal, 1985) failed because it was mathematically unsound (Bajzer et al., 1997). Clearly, a simple, biologically convincing, and mathematically sound argument for Gompertzian growth is still needed. The first goal of this paper is to develop such an argument. In that, we consider growth as a macrophenomenon, devoid of microscopic mechanisms except for the basic fact that a growing biological system consists of proliferating and quiescent cells.

Our point of departure is Gompertz' original idea. He developed his model from the insight that: “. . . the average exhaustion of man's power to avoid death [is] such that at the end of equal infinitely small intervals of time, he [loses] equal portions of his remaining power to oppose destruction which he had at the commencement of those intervals ...." (Gompertz, 1825, p. 518). In 1932, Winsor formulated this insight for biological growth: "In equal small intervals of time the organism looses equal proportions of its power to grow" (Winsor, 1932). Although this postulate is intuitively acceptable, the notion of the "power to grow" lacks biologic specificity. Probably because of that lack, the concept has not been developed much further; even Winsor himself used it only parenthetically to allude to the exponential behavior of the relative growth rate (Winsor, 1932).

In this paper, we examine Winsor's postulate of growth within the confines of our inference that the "power to grow" is quantified by the directly measurable growth fraction, the fraction of proliferating cells in the system. Here, the larger the growth fraction, the greater "the power" of the system to continue proliferating. On the basis of this interpretation of the "power to grow" and the notion that the increment in cellularity in a brief time interval is proportional to the number of proliferating cells and the time interval (first-order kinetics), we arrive at a plausible and mathematically rigorous derivation of Gompertzian growth. Scrutinizing this derivation, we realized that the first-order kinetics may be insufficient to account for the complexity of cell proliferation and loss. Consequently, our second goal was to investigate models still based on
Gompertzian growth fraction, but with more general kinetics, such as fractal kinetics. We found that fractal Gompertzian growth leads to Pütter's (1920) growth model, which encompasses other "classical" models (i.e. von Bertalanffy, the Bertalanffy-Richards, and the logistic model). In addition we discuss the self-similarity in Gompertzian growth (Bajzer 1999), and demonstrate that the recently invoked power-law growth (Hart et al., 1998) can be considered a limiting case of Gompertzian growth.

\section{GOMPERTZIAN GROWTH}

Along Winsor (1932), we assume that a biological system loses equal proportions of its "power to grow", $P(t)$, in equal small intervals of time, $\Delta t$. In mathematical terms, this assumption can be expressed as

$$
\frac{P\left(t_{1}+\Delta t\right)-P\left(t_{1}\right)}{P\left(t_{1}\right)}=\frac{P\left(t_{2}+\Delta t\right)-P\left(t_{2}\right)}{P\left(t_{2}\right)}<0 .
$$

Here $t_{1}$ and $t_{2}$ are arbitrary times elapsed since the onset of growth, and $P(t)$ is assumed to be a continuous function. From Equation (1) it follows that, for any time $t$,

$$
P(t+\Delta t) / P(t)-1=-\phi(\Delta t),
$$

where the function $\phi(\Delta t)>0$ does not depend on $t$. Assuming $P(t) \neq 0$ for any finite $t$, Equation (2) can be considered as a functional equation of the form $P(t+u)=P(t) f(u), f(u)=1-\phi(u)$. This equation has a trivial constant solution, and a solution given by the exponential function (Aczél, 1966; Bajzer, 1999):

$$
P(t)=P(0) e^{-b t}, \quad \phi(\Delta t)=1-e^{-b \Delta t} .
$$

Here $b=-\ln (1-\phi(1))>0$. It is noteworthy that, in deriving (3), we have not used the assumption that $\Delta t$ is small. This means that if Winsor's postulate holds for small time intervals, it also holds for any time interval.

At this point, the "power to grow" can be specified in terms of a biologically more meaningful quantity; we interpret the "power to grow" as growth fraction, the ratio of the number of proliferating cells $m(t)$ to 
the total number of cells $n(t)$ :

$$
P(t)=m(t) / n(t), \quad 0 \leq P(t) \leq 1
$$

One can consider $P(t)$ as the probability that cells proliferate at time $t$. Since at the inception of growth, the growth fraction is 1 (because the initial cell itself divides), from Equation (3) it follows that

$$
P(t)=e^{-b t}
$$

Assuming first order kinetics, the increment in cellularity $n(t)$ of the biological system within a brief time interval, $\Delta t$, is proportional to the number of proliferating cells and to $\Delta t$ :

$$
n(t+\Delta t)-n(t)=\kappa m(t) \Delta t .
$$

This equation implies that both the actual proliferation of cells and the loss of cells due to shedding and death in a brief time interval are proportional to the number of proliferating cells. For the rate of dying and shedding, this assumption might be unfounded and is being dealt with in the next section.

Finally, we assume that the size of the system is allometric to its cellularity,

$$
y(t)=C n^{a}(t) \quad a>0, \quad C>0 .
$$

Allometry takes into account the total tumor (or organism) volume (or mass) comprised of cells, extracellular space and fluids, vascular lumina, etc. When size is measured by cellularity, then obviously $C=1$ and $a=1$.

To obtain the rate of change in size, we combine Equations (4-7) to yield the differential equation ${ }^{\dagger}$

$$
y^{\prime}=k e^{-b t} y, \quad k=a \kappa .
$$

For $b \neq 0$, solution of Equation (8) is the Gompertz growth law that is most often written in

\footnotetext{
Equation (8) can be recast into a system of autonomous differential equations from the class of "synergistic and saturable systems" (Savageau, 1979):

$$
y^{\prime}=b x y, \quad x^{\prime}=-b x,
$$

where $x=k P / b$. Recently, we interpreted this system as "a particularly simple dynamics in which the growth rate and the growth fraction decay rate are governed by the same rate constant" (Bajzer et al., 1997). This interpretation is inaccurate because $x$ is only proportional, and not equal to the growth fraction $P$.
}

equivalent forms (Gompertz, 1825; Winsor, 1932; Norton, 1988):

$$
\begin{aligned}
y(t) & =p q^{r^{t}}=p \exp [-\exp (c-b t)] \\
& =y_{0} \exp [g(1-\exp (-b t))],
\end{aligned}
$$

where $y(0)=y_{0}>0$ and $b, g, c=\ln g, p=y_{0} \exp (g)$, $q=\exp (-g), r=\exp (-b)$ are characteristic parameters. For $b=0$, the solution of $(8)$ is exponential growth $y(t)=y_{0} \exp (k t)$ which corresponds to the maximal growth fraction, $P(t)=1$. Such solution cannot be obtained directly by choosing real values for parameters in any of the expressions (9); for example, in the last expression one cannot find real values of $y_{0}, g$, and $b$, so that this expression becomes a simple exponential function. $\ddagger$ Initial growth in many biological systems, including solid tumor appears exponential (Laird, 1966; Wheldon, 1988).

Traditionally, Gompertz function (9) was considered as the solution of (8), but also of the autonomous differential equation

$$
y^{\prime}=g_{1} y-b y \ln y, \quad g_{1}=b\left(g+\ln y_{0}\right) .
$$

which was often used in attempts to rationalize Gompertzian growth (Kendal, 1995; Xu, 1987; Gyllenberg and Webb, 1989; Calderon and Kwembe, 1991; Ling and He, 1993; Bajzer et al., 1996).

Gompertzian growth is unique among growth models because it can be viewed as a self-similar process. This becomes apparent when autonomous growth kinetics is represented by the functional, rather than differential, equation (Bajzer, 1999):

$$
y(t+s)=f(y(t), s)
$$

Here growth kinetics is defined by a continuous function $f(y, s)$ that relates the size $y=y(t)$ of the system at time $t$ to its size $s$ time units later. If this function is self-similar in $y$, the solution of (11) is the Gompertz function or exponential function (Bajzer, 1999). Self-similarity, in its general meaning, implies invariance to scaling ( $c f$. Bassingthwaighte et al., 1994). In our case, it is assumed that, for

\footnotetext{
F However, if $g$ is assumed to be dependent on $b$, i.e., $g=k / b$ where $k$ does not depend on $b$, then $\lim _{b \rightarrow 0} y=y_{0} \exp (k t)$.
} 
each $s$ and for any scaling factor $\lambda>0, f(y, s)$ is a homogeneous function of the degree $\beta(s)>0$ with respect to variable $y$ :

$$
f(\lambda y, s)=\lambda^{\beta(s)} f(y, s) .
$$

According to Mandelbrot (1983), for $\beta(s) \neq 1$ such function is self-affine with respect to exponent $\beta(s)$ and to zero focal value; for $\beta(s)=1$, it is a selfsimilar function in the strict sense.

Equation (12) can be viewed as a functional equation for $f$ that can be solved by setting $y=1$ to yield $f(y, s)=\alpha(s) y^{\beta(s)}, \alpha(s)=f(1, s)$. Together with (11), this defines the following functional equation in $y$ :

$$
y(t+s)=\alpha(s)[y(t)]^{\beta(s)} .
$$

Solution of this equation is the Gompertz function (9) with $\beta(s)=\exp (-b s)=r^{s}$ and $\alpha(s)=p^{1-\beta(s)}$ (Bajzer, 1999). For $b=0$, it follows that $\beta(s)=1$, which implies strict self-similarity. In this case, the solution of (13) is an exponential function.

\section{GOMPERTZIAN GROWTH WITH FRACTAL KINETICS}

The essence of Gompertzian growth is the exponential decay of the "power to grow" or, in our interpretation, of the growth fraction. Staying with this interpretation, we examine Gompertzian growth when cell proliferation does not obey the first order kinetics. Rather, the change in cellularity of the system is proportional to the power of the number of proliferating cells, i.e., growth obeys fractal kinetics ( $c f$. Kopelman, 1988; Ahmed, 1993; Dewey, 1997):

$$
n(t+\Delta t)-n(t)=\kappa m^{d}(t) \Delta t, \quad d>0 .
$$

In Equation (14), all change in cellularity is still related to the number of proliferating cells at any given time, but changes due to cell death and shedding can be accommodated with more flexibility allowed by the exponent $d$ [compare to Equation (6)].
Now, Equations (14) and (7), together with (4) and (5), yield

$$
y^{\prime}=k e^{-\gamma t} y^{\delta}, \quad y(0)=y_{0}
$$

with

$$
k=C^{1-\delta} a \kappa, \quad \delta=(d+a-1) / a, \quad \gamma=b d>0 .
$$

Equation (15) describes growth in terms of Winsor's postulate, of our substituting "power to grow" for the growth fraction, and of fractal kinetics (14).

For $\delta \neq 1$, Equation (15) can be solved by the standard separation of variables yielding

$$
y^{1-\delta}=k(1-\delta) / \gamma+y_{0}^{1-\delta}-k(1-\delta) e^{-\gamma t} / \gamma
$$

This equation implies that for all $t>0$ the real solution exists only if the constant term in (17) is positive, i.e. if

$$
K \equiv k(1-\delta) / \gamma+y_{0}^{1-\delta}>0 .
$$

The solution of (15) assumes the form:

$$
y(t)=A\left(1+B e^{-\uparrow}\right)^{D},
$$

where

$$
\begin{gathered}
D=1 /(1-\delta), \quad B=k /\left(\gamma_{1} K\right), \quad A=K^{D}, \\
\gamma_{1}=\gamma /(1-\delta) .
\end{gathered}
$$

Function (19) is known as Pütter's growth function (Pütter, 1920; cf. Lebeau et al., 1986; Jolicoeur and Pirlot, 1988). Pütter's function encompasses also the von Bertalanffy growth model and the von Bertalanffy-Richards model. Indeed, for $D<0(\delta>$ 1 ), it follows that $B>0$. Then, from Equation (19) one obtains

$$
e^{-\gamma t}=\left[(y / A)^{1 / D}-1\right] / B
$$

which, reintroduced into Equation (15), yields the autonomous differential equation

$$
y^{\prime}=\gamma_{1} y\left[1-(y / A)^{-1 / D}\right]
$$

known as the von Bertalanffy-Richards growth model (cf. Turner et al. 1976; Bajzer et al., 1996). For $D=$ $-1,(\delta=2)$, this equation becomes the standard logistic equation with the carrying capacity $A$. 
Analogously, for $D>0,(\delta<1)$, one obtains the generalized von Bertalanffy model ( $c f$. Bajzer et al., 1997),

$$
y^{\prime}=b_{1} y^{\delta}-\gamma_{1} y,
$$

with $b_{1}=k /|B|$. For $\delta=2 / 3$ (which implies $D=3$ in $(18)$ ), this becomes the standard "surface rule" von Bertalanffy model based on the notion that volume buildup is proportional to surface area.

For $\delta=1$, the function (19) with (20) is not defined. However, solution of Equation (15) is the original Gompertz function (9). It is noteworthy that $\delta=1$ does not imply fractal kinetics, because $(d+a-$ 1) $/ a=1$ yields $d=1$. In other words, the original Gompertz model is devoid of fractal kinetics.

On the basis of the preceding discussion, we conclude that Equation (15), Gompertzian growth with fractal kinetics, actually describes the logistic and the von Bertalanffy models and their generalizations. For $\delta \neq 1$, Equation (15) is equivalent to autonomous differential Equations (22) and (23) which, together with the original Gompertz model [(15) with $\delta=1$, or (10)], constitute what we named the generalized von Bertalanffy-logistic model (Marušić et al., 1994a). It is interesting that in a recent study of growth of primary breast cancer, Equation (15) described the data most adequately with $\delta=5 / 4$ (Spratt et al., 1993) indicating that in this case growth was described by the von Bertalanffy-Richards law. In a study of tumor xenografts (Michelson et al., 1987), the BertalanffyRichards model (termed "modified Verhulst model") yielded $\delta=1.005$, very close to the Gompertz model $(\delta=1)$; in one case, $\delta=1.5$ was found.

\section{POWER-LAW GROWTH, A LIMITING CASE OF GOMPERTZIAN GROWTH}

Recently Hart et al. (1998) obtained the growth law for primary breast cancer from distribution of tumor sizes determined by mammography. The authors found that the Gompertz model and the logistic model could not describe this distribution as well as the power-law growth (Mendelsohn, 1963) that was previously used to describe the growth of mammary tumors (Dethlefsen et al., 1968).
Here we note that the power-law growth, defined by

$$
y^{\prime}=k y^{\eta}, \quad y(0)=y_{0}>0, \quad k>0, \quad \eta>0 .
$$

can be considered a limiting case of Gompertzian growth. Indeed, in the limiting case $b \rightarrow 0$, which implies $P(t) \rightarrow 1$, Equation (15) reduces to (24). When $P(t)=1$ for all $t$, it means that the growth fraction is constant, contrary to Gompertzian notion of diminution of the "power to grow".

Consistent with power-law growth (24) and fractal kinetics (14), one can also assume that the growth fraction is inversely proportional to the power of the number of cells:

$$
P(t) \propto 1 / n^{\varepsilon}(t), \quad \varepsilon>0,
$$

Simple algebra reveals that $\eta$ in (24) is related to exponents $a>0$ in (7) and $d>0$ in (14) as $\eta=$ $d(1-\varepsilon) / a+1-1 / a$. Thus, the power-law growth could be considered consequence of fractal kinetics or first order-kinetics $(d=1)$ and of the growth fraction that decreases with cellularity as in (25). Despite the decrease in growth fraction, the overall growth given by (24) is an ever increasing function without asymptote:

$$
y(t)=\left[(1-\eta) k t+y_{0}^{1-\eta}\right]^{1 /(1-\eta)}
$$

This function is defined for all $t$, when $\eta<1$, and only for a limited time if $\eta>1$. Hart et al. (1998) found $\eta \approx 0.5$ and Dethlefsen et al. (1968) also found $\eta$ smaller than one.

\section{DISCUSSION}

We have used the notion of the "power to grow" and interpreted it as growth fraction, a biologically meaningful quantity that is easy to perceive and measure. This interpretation allowed us to separate conceptually the growth fraction from growth kinetics in the Gompertz model, resulting in the demonstration that inherent in Gompertzian growth is the elementary natural principle of cell division and its diminishing probability as the system grows. Another important 
consequence of the separation of the growth fraction from growth kinetics is the opportunity to analyze Gompertzian growth under different kinetic regimens. The classical Gompertz model implies first-order kinetics as inherent in human reproduction. Yet, volume growth of organisms and tumors takes place in fractal space of noninteger number of dimensions ( $c f$. Kopelman, 1988; Vicsek, 1992; Nonnenmacher et al., 1993). We found that Gompertzian growth, generalized for $\delta \neq 1$, includes other well known empirical deterministic models such as the logistic model $(\delta=$ 2 ), models of von Bertalanffy $(\delta<1 ; \delta=2 / 3)$ and the von Bertalanffy-Richards model $(\delta>1)$. Thus, for biologic (organism, tumor) growth, the originative Gompertz model is just a special case of Gompertzian growth that is based on fractal kinetics. In practical terms, when, for example, the Gompertz function does not fit the data and the von Bertalanffy-Richards function does, it suggests that the cell population (or volume) changes size within a fractal space.

Beyond the new insights, extension of the Gompertz model into Gompertzian fractal growth provides new opportunities to study the natural history of organisms, tissues and tumors. For example, from experimentally determined values of the growth fraction and from measured growth curves, it is now possible to deduce the fractal signature of the growth space within a biological system.

We noted that the power-law growth (Mendelsohn, 1963; Hart et al., 1998) can be interpreted as a limiting case of Gompertzian growth with growth fraction constant. However, this is not the only interpretation of the power-law growth. When we separated conceptually the growth fraction from the kinetic equation, we found that the power-law growth is consistent also with a diminishing growth fraction that is proportional to the negative power of the total cell number. Such growth fraction behavior could be essentially different from the exponential growth fraction decay that characterizes Gompertzian growth.

\section{Acknowledgements}

The stem Cell Laboratory gratefully acknowledges the generous support from Mrs. Adelyn
L. Luther, Singer Island, Florida. The authors thank Dr. Franklyn G. Prendergast for his continuing mentorship, interest and support.

\section{References}

Aczél, J. (1966). Lectures on Functional Equations and their Applications, Academic Press, New York.

Adam, J. A. and Bellomo, N., eds. (1997). A Survey of Models for Tumor-Immune System Dynamics, Birkhäuser, Boston.

Agur, Z. (1998). Resonance and anti-resonance in the design of chemotherapeutic protocols. Journal of Theoretical Medicine, $\mathbf{1}$, $237-245$.

Agur, Z., Arnon, R. and Schechter, B. (1992). Effect of the dosing interval on myelotoxicity and survival in mice treated by cytarabine. European Journal of Cancer, 28A, 1085-1092.

Ahmed, E. (1993). Fractals and chaos in cancer models. International Journal of Theoretical Physics, 32, 353-355.

Asachenkov, A., Marchuk, G., Mohler, R. and Zuev, S. (1994). Disease Dynamics, Birkhäuser, Boston, 198-316.

Akanuma, A. (1978). Parameter analysis of Gompertzian function growth model in clinical tumors. European Journal of Cancer, 14, 681-688.

Bajzer, Ž. (1999). Gompertzian growth as a self-similar and allometric process. Growth, Develpment and Aging, 63, 3-11.

Bajzer, Ž., Marušić, M. and Vuk-Pavlovic, S. (1996). Conceptual frameworks for mathematical modelling of tumor growth dynamics. Mathematical and Computer Modelling, 23, 31-46.

Bajzer, Ž., Pavelić, K. and Vuk-Pavlović, S. (1984). Growth selfincitement in murine melanoma B16: a phenomenological model. Science, 225, 930 932.

Bajzer, Ž. and Vuk-Pavlović, S. (1990). Quantitative aspects of autocrine regulation in tumors. Critical Reviews in Oncogenesis, 2. 53-73.

Bajzer, Ž., Vuk-Pavlović, S. and Huzak, M. (1997). Mathematical modelling of tumor growth kinetics. In A Survey of Models for Tumor-Immune System Dynamics (eds. J. A. Adam and N. Bellomo), Birkhäuser, Boston, pp. 89-132.

Bassingthwaighte, J. B., Liebovitch, L. S. and West, G. B. (1994). Fractal Physiology. Oxford University Press, New York, 16-19.

Bassukas, I. D. (1994a). Comparative Gompertzian analysis of alterations of tumor growth. Cancer Research, 54, 4385-4392.

Bassukas, I. D. (1994b). Gompertzian re-evaluation of the growth patterns of transplantable mammary tumors in sialoadenectomized mice. Cell Proliferation, 27, 201-211.

Bassukas, I. D. and Maurer-Schultze, B. (1988). The recursion formula of the Gompertz function: a simple method for the estimation and comparison of tumor growth curves. Growth, Develpment and Aging, 52, 113-122.

Begall, S. (1997). The application of the Gompertz model to describe body growth. Growth, Develpment and Aging, 61, 61-67.

von Bertalanffy, L. (1957). Quantitative laws in metabolism and growth. Quaterly Reviews in Biology, 32, 217-231.

Calderón, C. P. and Kwembe, T. A. (1991). Modeling tumor growth. Mathematical Biosciences, 103, 97-114.

Cameron, D. A. (1997). Mathematical modeling of the response of breast cancer to drug therapy. Journal of Theoretical Medicine, $1,137-151$.

Cojocaru, L. and Agur, Z. (1992). A theoretical analysis of interval drug dosing for cell-cycle-phase-specific drugs. Mathematical Biosciences, 109, 85-97. 
Dethlefsen, L. A., Prewitt and Mendelsohn, M. L. (1968). Analysis of tumor growth curves. Journal of the National Cancer Institute, 40, 389-405.

Dewey, T. G. (1997). Fractals in Molecular Biophysics. Oxford University Press, Oxford.

Durbin, P. W., Jeung, N., Williams, M. H. and Arnold, J. S. (1967). Construction of a growth curve for mammary tumors of the rat. Cancer Research, 27, 1341-1347.

Frenzen, C. L. and Murray, J. D. (1986). A cell kinetics justification for Gompertz' equation. SIAM Journal of Applied Mathematics, 46, 614-629.

Gompertz, B. (1825). On the nature of the function expressive of the law of human mortality, and on a new mode of determining the value of life contingencies. Philosphical Transactions of the Royal Society, London, 115, 513-585.

Gratton, R. J., Appleton, D. R. and Alwiswasy, M. K. (1978). The measurement of tumor growth rates. Biomathematics and Cell Kinetics, Elsevier/North-Holland Biomedical Press, 325-332.

Gyllenberg, M. and Webb, G. F. (1989). A nonlinear structured population model of tumor growth with quiescence. Growth, Development and Aging, 53, 25-33.

Hart, D., Shochat, E. and Agur, Z. (1998). The growth law of primary breast cancer as inferred from data from mammography screening trials. British Journal of Cancer, 78, 382-387.

Jolicoeur, P. and Pirlot, P. (1988). Asymptotic growth and complex allometry of the brain and body in the white rat. Growth, Development and Aging, 52, 3-10.

Kendal, W. S. (1985). Gompertzian growth as a consequence of tumor heterogeneity. Mathematical Biosciences, 73, 103-107.

Kopelman, R. (1988). Fractal reaction kinetics. Science, 241, $1620-1626$.

Laird, A. K. (1964). Dynamics of tumor growth. British Journal of Cancer, 18, 490-502.

Laird, A. K. (1966). Dynamics of embryonic growth. Growth, 30, $349-363$.

Laird, A. K., Tyler, S. A. and Barton, A. D. (1965). Dynamics of normal growth. Growth, 29, 233-248.

Lebeau, B., Jolicoeur, P., Pageau, G. and Crossman, E. J. (1986). Asymptotic growth, egg production and trivariate allometry in Esox Masquinongy Mitchill. Growth, 50, 185-200.

Ling, Y. and He, B. (1993). Entropic analysis of biological growth models. IEEE Transactions of Biomedical Engineering, $1193-1200$.

Makany, R. (1991). A theoretical basis for Gompertz's curve. Biometrics Journal, 33, 121-128.

Mandelbrot, B. B. (1983). The Fractal Geometry of Nature. Freeman, New York, 349-350.

Martin, R. B., Fisher, M. E., Minchin, R. F. and Teo, K. L. (1990). A mathematical model of cancer chemotherapy with an optimal selection of parameters. Mathematical Biosciences, 99 , $205-230$.

Marušić, M., Bajzer, Ž., Freyer, J. P. and Vuk-Pavlović, S. (1994a). Analysis of growth of multicellular tumor spheroids by mathematical models. Cell Proliferation, 27, 73-94.

Marušić, M., Bajzer, Ž., Vuk-Pavlović, S. and Freyer, J.P. (1994b). Tumor growth in vivo and as multicellular spheroids compared by mathematical models. Bulletin of Mathematical Biology, 56, 617-631.

Mendelsohn, M. L. (1963). Cell proliferation and tumour growth. In Cell Proliferation (eds. L.F. Lamerton and R.J.M. Fry), Blackwell, London, pp. 190-210.

Michelson, S., Glicksman, A. S. and Leith, J. T. (1987). Growth in solid heterogeneous colon adenocarcinomas: comparison of simple logistical models. Cell and Tissue Kinetics, 20, 343-355.

Michelson, S. and Leith, J. T. (1996). Host response in tumor growth and progression. Invasion and Metastasis, 16, 235-246.
Nonnenmacher, T. F., Losa, G. A. and Weibel, E. R., eds. (1993) Fractals in Biology and Medicine, Birkhäuser, Basel.

Norton, L. (1988). A Gompertzian model of human breast cancer growth. Cancer Research, 48, 7067-7071.

Norton, L., Simon, R., Brereton, H. D. and Bogden, A. E. (1976). Predicting the course of Gompertzian growth. Nature, 264 , $542-545$.

Olea, N., Villalobos, M., Nuñez, M. I., Elvira, J., Ruiz de Amodóvar, J. M. and Pedraza, V. (1994). Evaluation of the growth rate of MCF-7 breast cancer multicellular spheroids using three mathematical models. Cell Proliferation, 27, 213-223.

Panetta, J. C. (1996). A mathematical model of periodically pulsed chemotherapy: tumor recurrence and metastasis in a competitive environment. Bulletin of Mathematical Biology, 58, 425-447.

Parfitt, A. M. and Fyhrie, D. P. (1997). Gompertzian growth curves in parathyroid tumors: further evidence for the set-point hypothesis. Cell Proliferation, 30, 341-349.

Pütter, A. (1920). Studien über physiologische Ähnlichkeit. VI. Wachstumsähnlichkeiten. Pfügers Archiv gesamte Physiologie, 180, $298-340$.

Savageau, M. A. (1979). Allometric morphogenesis of complex systems: A derivation of the basic equations from first principles. Proceedings of the National Academy of Sciences, 76, 5413-5417.

Shochat, E., Hart, D. and Agur, Z. (1999). Using computer simulations for evaluating the efficacy of breast cancer chemotherapy protocols. Journal of Mathematical Modelling and Methods in Applied Sciences, 9, 599-615.

Steel, G. G. (1977). Growth Kinetics of Tumors. Clarendon Press, Oxford.

Simpson-Herren, L. and Lloyd, H. (1970). Kinetic parameters and growth curves for experimental tumor systems. Cancer Chemotherapy Reports, 54, 143-174.

Spratt, J. A., von Fournier, D., Spratt, J. S, and Weber, E. E. (1993). Decelerating growth and human breast cancer. Cancer, 71, 2013-2019.

Sullivan, P. W. and Salmon, S. E. (1972). Kinetics of tumor growth and regression in IgG multiple myeloma. Journal of Clinical Investigation, 51, 1697-1708.

Swan, G. W. (1990). Role of optimal control theory in cancer chemotherapy. Mathematical Biosciences, 101, 237-284.

Turner, Jr., M. E., Bradley, Jr., E. L., Kirk, K. A. and Pruitt, K. M. (1976). A theory of growth. Mathematical Biosciences, 29, 367-373.

Vaidya, V. G. and Alexandro, Jr., F. J. (1982). Evaluation of some mathematical models for tumor growth. International Journal of Biomedical Computing, 13, 19-35.

Vicsek, T. (1992). Fractal Growth Phenomena, 2nd ed., World Scientific Pub., Singapore.

Webb, G. F. (1992). A nonlinear cell population model of periodic chemotherapy treatment. In Recent Trends in Ordinary Differential Equations, Series in Applicable Analysis, World Scientific Pub., Singapore, pp. 569-583.

Weymouth, F. W., McMillin, H. C. and Rich, W. H. (1931). Latitude and relative growth in the razor clam. Siliquia Patula, Joumal of Experimental Biology, 8, 228-249.

Wheldon, T. E. (1988). Mathematical Models in Cancer Research, Adam Hilger, Bristol.

Winsor, C. P. (1932). The Gompertz curve as a growth curve. Proceedings of the National Academy of Sciences, 18, 1-8.

Witten, M. (1985). A retum to time, cells, systems, and aging: III. Gompertzian models of biological aging and some possible roles for critical elements. Mechanisms of Ageing and Development, 32, 141-177. 
Wosilait, W. D., Luecke, R. H. and Young, F. W. (1992). A mathematical analysis of human embryonic and fetal growth data. Growth, Development and Aging, 56, 249-257.
Xu, X. (1987). The biological foundation of the Gompertz model. International Journal of Biomedical Computing, 20, $35-39$. 


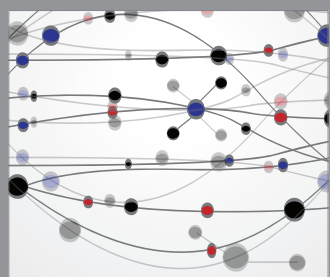

The Scientific World Journal
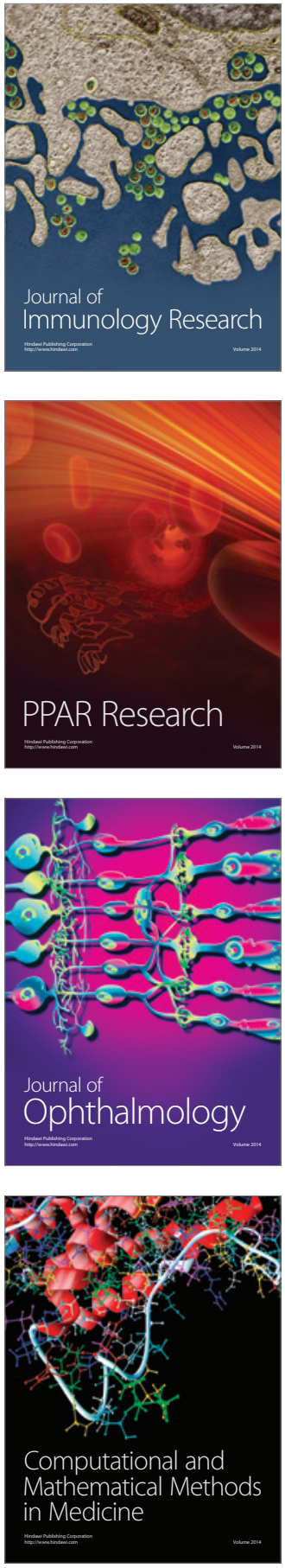

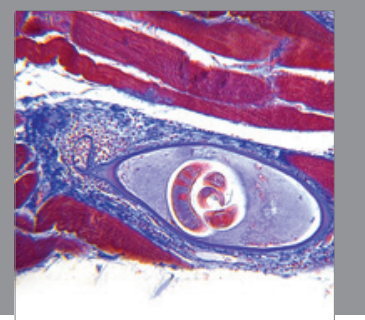

Gastroenterology

Research and Practice
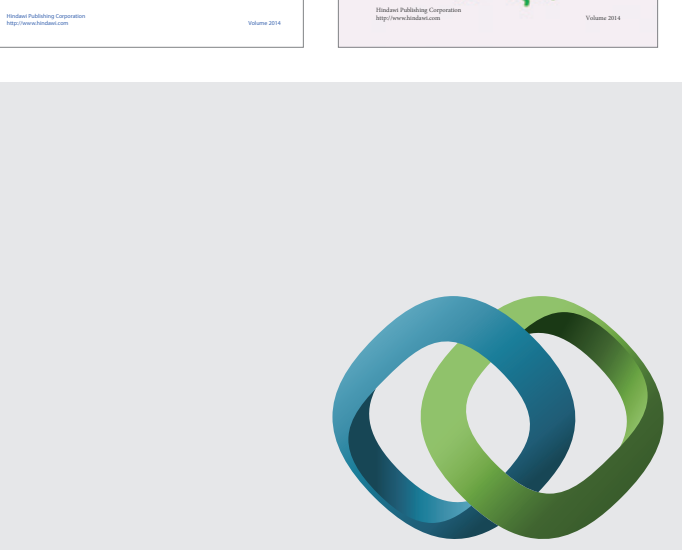

\section{Hindawi}

Submit your manuscripts at

http://www.hindawi.com
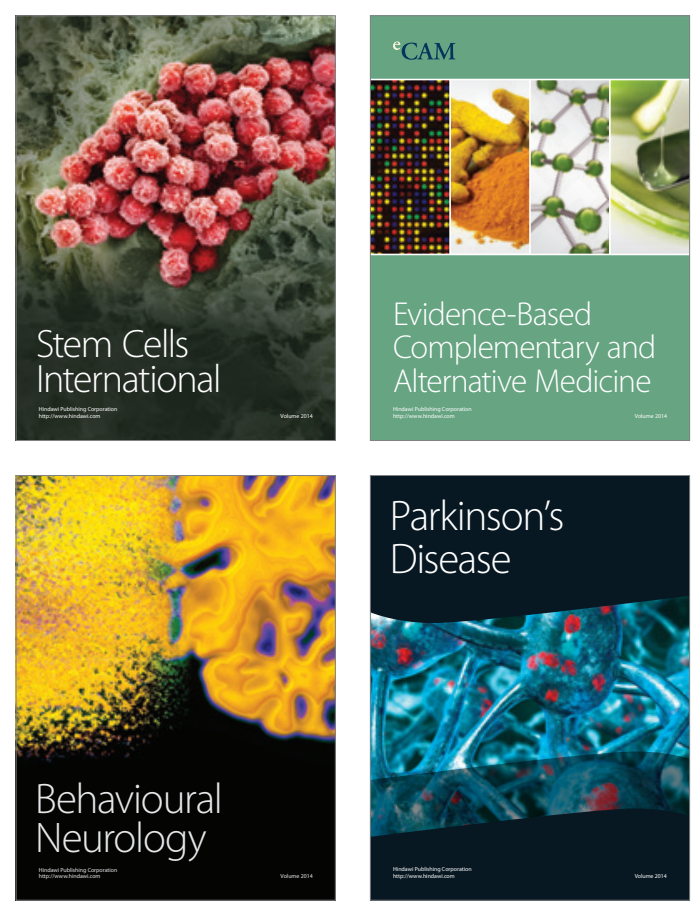

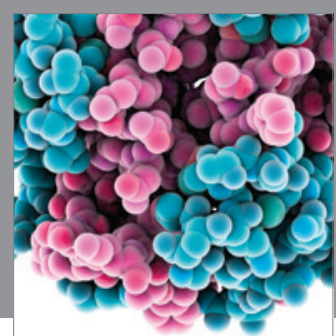

Journal of
Diabetes Research

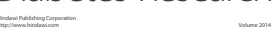

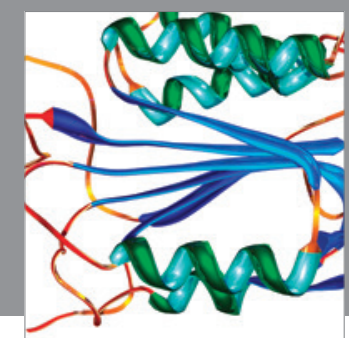

Disease Markers
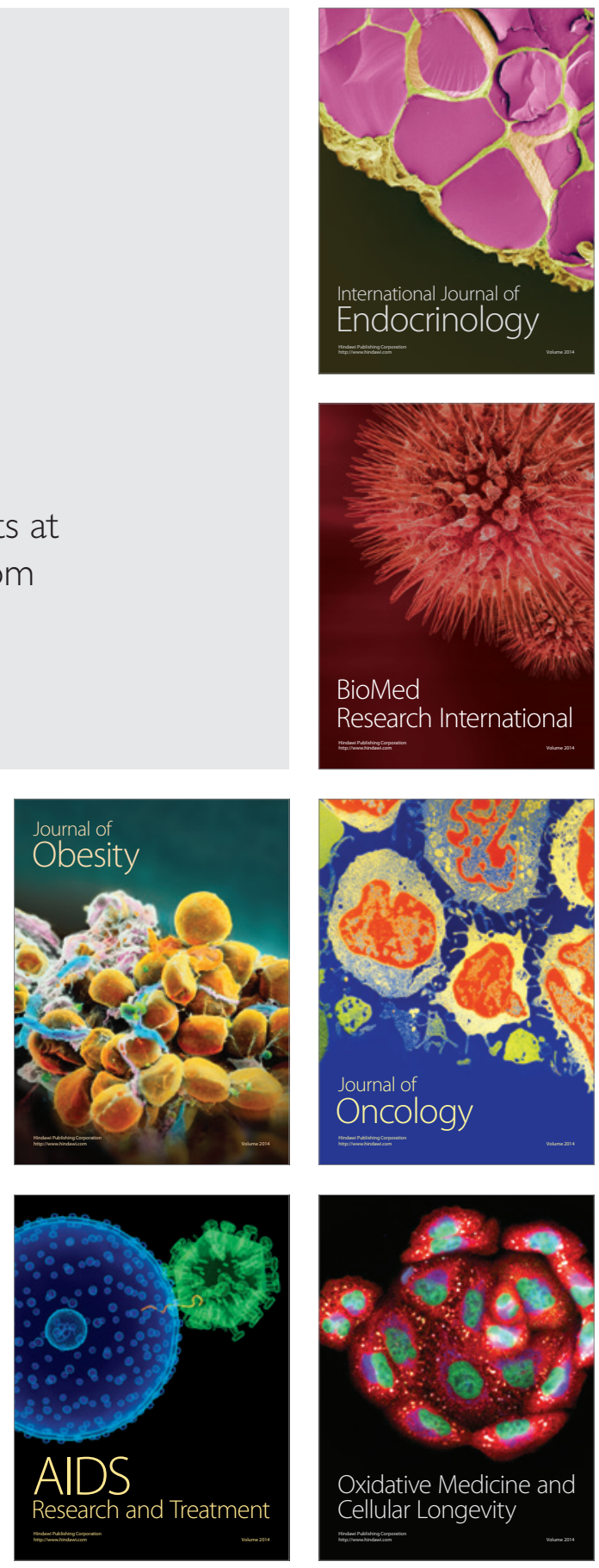